\title{
Flexibler, amperometrischer Biosensor basierend auf der Seide des Seidenspinners Bombyx mori
}

Denise Molinnus ${ }^{1,2}$, Kevin A. Janus ${ }^{1}$, Aleksander Drinic ${ }^{3}$, Heiko Iken ${ }^{1}$, Nadja Kröger ${ }^{4}$, Max Zinser ${ }^{4}$, Ralf Smeet ${ }^{5}$, Marius Köpf ${ }^{3}$, Alexander Kopp ${ }^{3}$, Michael Keusgen ${ }^{6}$, Michael J. Schöning ${ }^{1,7}$

\author{
${ }^{1}$ Institut für Nano- und Biotechnologien (INB), FH Aachen, Jülich, Deutschland \\ ${ }^{2}$ Klinik für Operative Intensivmedizin und Intermediate Care, Innovationszentrum Digitale Medizin, Medizini- \\ sche Fakultät RWTH Aachen, Aachen, Deutschland \\ ${ }^{3}$ Fibrothelium $\mathrm{GmbH}$, Aachen, Deutschland \\ ${ }^{4}$ Abteilung für plastische, rekonstruktive und ästhetische Chirurgie, Universitätskrankenhaus Köln, Köln, \\ Deutschland \\ ${ }^{5}$ Klinik für Mund-, Kiefer- und Gesichtschirurgie, Abteilung für regenerative orofaziale Medizin, Universitäts- \\ klinikum Hamburg-Eppendorf, Hamburg, Deutschland \\ ${ }^{6}$ Institut für Pharmazeutische Chemie, Philipps Universität Marburg, Marburg, Deutschland \\ ${ }^{7}$ Institut für Biologische Informationsprozesse (IBI-3), Forschungszentrum Jülich, Jülich, Deutschland \\ Kontakt:molinnus@fh-aachen.de
}

\section{Einleitung}

Biokompatible und biodegradierbare Materialien haben ein enormes Einsatzpotential im Bereich der Medizintechnik, z.B. bei der Entwicklung von biomedizinischen Sensoren, Drug Delivery-Sytemen oder beim Tissue Engineering [1]. Auf Grund ihrer einzigartigen Charakteristika, d.h. insbesondere die gute mechanische Stabilität bei gleichzeitig geringem Risiko einer Auslösung von Entzündungsreaktionen im Körper, ist bei vielen medizinischen Fragestellungen die Verwendung eines solchen Materials von hohem Interesse.

Ein vielversprechender Ansatz für die Entwicklung eines Biosensors für den Einsatz in der Medizin ist die Verwendung von Seide des Seidenwurms Bombyx mori. Seide ist eine altbekannte tierische Naturfaser, die durch ihre einstellbare biologische Abbaubarkeit und ihre herausragenden mechanischen Eigenschaften eine Renaissance in der modernen Technik erfahren hat [2]. Das Fibroinprotein ist wasserlöslich und kann daher in verschiedenen Strukturen (z.B. als Schwamm, Membran, Gel, Pulver) hergestellt werden [3]. Der Degradationsprozess der Seide ist unter anderem von der Architektur und der Morphologie der regenerierten Proteinstrukturen abhängig und wird über proteolytische Enzyme wie Kollagenase, Keratinase und Elastase gesteuert [4-6].

In der Literatur werden bereits verschiedene Sensoren basierend auf Seidenfibroin beschrieben. Mittels Graphen-Elektrode, aufgebracht auf einem Zahn, konnten beispielsweise Staphilococcus aureus-Infektionen und andere Bakterien nachgewiesen werden [2].

In dieser Arbeit wurde ein „Proof-of-Concept"-Biosensorexperiment erarbeitet, um das zukünftige Potential der seidenbasierten Sensorik zu verdeutlichen: Durch die geschickte Kombination von Dünnschicht- und Dickschichttechnologien, zur Herstellung von Platin-Arbeits- und Platin-Gegenelektroden bzw. Ag/AgCl-Quasi-Referenzelektroden, wurden flexible Biosensoren auf Basis von biologisch abbaubarem Seidenfibroin entwickelt. Die Sensoren, verkapselt in biokompatiblem Silikon, wurden am Beispiel eines Glukosebiosensors mit dem Enzym Glukose-Oxidase untersucht. Die Funktionsfähigkeit des Sensors konnte durch erfolgreiche Glukosemessungen in Puffer- und in Ringerlösung demonstriert werden. Degradationstests des Fibroinsubstrats mit Platin-Elektroden wurden ebenfalls durchgeführt.

\section{Methoden und Materialien}

Für die Herstellung des Fibroinsubstrats wurden Seiden-Kokons (Bombyx mori) zur Proteinextraktion (Cantiere della Provvidenza, Italy) genutzt. Glycerin und Ethanol wurden von VWR International (Belgien) gekauft. Natriumcarbonat wurde von AppliChem $\mathrm{GmbH}$ (Deutschland) erworben und Calciumchlorid von Merck KGaA (Deutschland).

Zur Herstellung der Enzymmembran mittels Quervernetzer wurde Glutaraldehyd, Rinderserum-Albumin (BSA), Glycerin, das Enzym Glukose-Oxidase (GOX, EC 1.4.4., von Aspergillus niger) sowie die Pufferkomponenten (Mononatriumphosphat und Dinatriumphosphat) von Sigma-Aldrich (USA) bestellt. Ringerlösung $(8,6 \mathrm{~g} / \mathrm{L} \mathrm{NaCl}, 0,3 \mathrm{~g} / \mathrm{L} \mathrm{KCl}, 0,33 \mathrm{~g} / \mathrm{L} \mathrm{CaCl} 2$ - $2 \mathrm{H}_{2} \mathrm{O}$ ) wurde von Bernburg (Deutschland) gekauft. Zur Herstellung der Quasi-Referenzelektrode wurde eine $\mathrm{Ag} / \mathrm{AgCl}$-Paste der Firma DuPont (Großbritannien) erworben. Zur Verkapselung wurde das biokompatible Zweikomponenten-Silikon ECOFLEX 0030 von KauPo Plankenhorn e.K. (Deutschland) gekauft. Diese beiden Komponenten wurden in einem 1:1 Verhältnis gemischt und für 3 min vor Nutzung entgast.

Die detaillierte Herstellung des Fibroinsubstrats kann in [7] nachgelesen werden. Zusammengefasst wurde die 4\% (w/v) Fibroinlösung mit Glycerol/Ethanol $(50: 50(\mathrm{v} / \mathrm{v}))$ gemischt, um eine 50\% Fibroinzusam- 
mensetzung zu erhalten. Zur Herstellung der Substratstruktur wurde die Fibroinzusammensetzung auf einem Silizium-Wafer als Träger aufgegossen und für $24 \mathrm{~h}$ bei $21^{\circ} \mathrm{C}$ getrocknet. Das finale Fibroinsubstrat hatte eine Dicke von $200 \mu \mathrm{m}$, auf das dann die Sensorstrukturen abgeschieden werden konnten.

Der Sensorchip, bestehend aus Arbeits-, Referenzund Gegenelektrode, wurde mittels Dick- und Dünnschichttechnologien hergestellt. Die Platin-Arbeitselektrode und -Gegenelektrode wurden unter Reinraumbedingungen mittels Dünnschichttechnologie prozessiert. Zunächst wurde eine $20 \mathrm{~nm}$ Titanschicht mittels Elektronenstrahlverdampfung aufgebracht, gefolgt von einer $200 \mathrm{~nm}$ dicken Platinschicht mittels Schattenmaske. Der finale Wafer mit den $15 \mathrm{~mm}$ x $15 \mathrm{~mm}$ Chips ist in Abb. 1 a) dargestellt. Jeder Sensorchip besteht aus vier Platinelektroden, wobei zwei als Arbeits- und zwei als Gegenelektrode für die amperometrischen Messungen genutzt wurden. Die Platin-Arbeitselektrode wurde mit einer Enzymmembran zur Detektion von Glukose modifiziert. Der Enzymmembrancocktail bestand aus $20 \mu \mathrm{L}$ Glukose-Oxidase $(4 \mathrm{U} / \mu \mathrm{L}), 40 \mu \mathrm{L}$ BSA (10\%) und $40 \mu \mathrm{L}$ Glutaraldehyd $(2 \%) /$ Glycerin (10\%). Alle Komponenten wurden unmittelbar vor Gebrauch zubereitet und in Phosphatpuffer (PBS, $10 \mathrm{mM}, \mathrm{pH} 7,4$ ) gelöst. Der finale Enzymcocktail wurde mit den folgenden Volumenverhältnissen hergestellt: 1-2-2 (Enzym-BSA-Glutaraldehyd/Glycerin) [8]. Es wurden $4 \mu \mathrm{L}$ mit 3,2 $\cup$ GOX des Enzymmembrancocktails auf die Platin-Arbeitselektrode (Durchmesser von $2 \mathrm{~mm}$ ) pipettiert und für $2 \mathrm{~h}$ bei Raumtemperatur getrocknet.

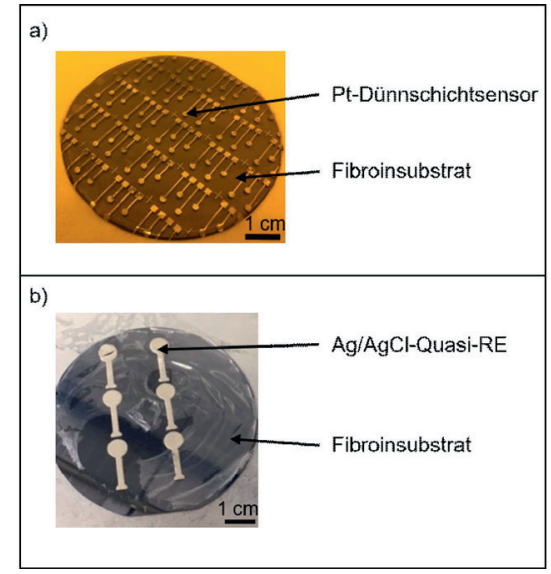

Abb. 1: Lichtmikroskopieaufnahmen a) des 4-Zoll Fibroinsubstrats mit den Platin (Pt)-Dünnschichtelektroden hergestellt mittels Dünnschichttechnologie und b) des 4-Zoll Fibroinsubstrats mit den Ag/AgCl-Quasi-Referenzelektroden hergestellt mittels Dickschichttechnologie.

Die Ag/AgCl-Quasi-Referenzelektrode wurde mittels Dickschichttechnologie hergestellt. Die $\mathrm{Ag} / \mathrm{AgCl}-$ Paste wurde über eine Schablone auf das Fibroin mittels Dickschichtdrucker (Essemtec AG, Switzerland) gerakelt, wobei Strukturen mit einer Dicke von $20 \mu \mathrm{m}$ entstanden sind (s. Abb. 1 b)). Die gedruckten Sensoren mussten für $2 \mathrm{~h}$ bei $60^{\circ} \mathrm{C}$ in einem herkömmlichen Ofen ausgehärtet werden. Jede QuasiReferenzelektrode (Durchmesser von $7 \mathrm{~mm}$ ) wurde mittels Skalpell in eine Größe von $10 \mathrm{~mm}$ x $20 \mathrm{~mm}$ zurechtgeschnitten.

Sowohl die Pt-Arbeits- und -Gegenelektroden als auch die Ag/AgCl-Quasi-Referenzelektroden wurden mittels Kontaktierdrähten mit Silberleitlack (Ferrero $\mathrm{GmbH}$, Deutschland) kontaktiert. Beide Chip-Komponenten, Sensor mit integrierter Arbeits- und Gegenelektrode, sowie die Quasi-Referenzelektrode, wurden mit den jeweiligen Rückseiten aufeinander geklebt (siehe Abb. 2). Hierzu wurde das biokompatible Silikon verwendet. Um die Biokompatibilität des ganzen Biosensorchips zu gewährleisten, wurde, bis auf die Messflächen, der Sensorchip mit dem gleichen biokompatiblen Silikon verkapselt.

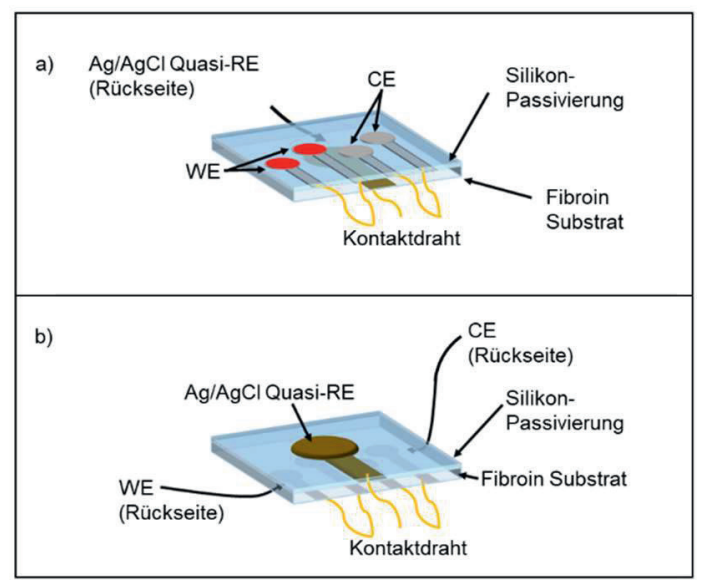

Abb. 2: Schematische Darstellung des biokompatiblen Glukosebiosensors, hergestellt mittels Dünn- und Dickschichttechnologien mit Pt-Arbeits (WE)- und -Gegenelektroden (CE) auf der einen Seite und einer $\mathrm{Ag} / \mathrm{AgCl}-$

Quasi-Referenzelektrode (RE) auf der anderen Seite.

Zur Charakterisierung des hergestellten Glukosebiosensors mit der Dreielektrodenanordnung, unter der Verwendung von Fibroin als Substrat, wurde der Sensorchip an einen Potentiostaten (PalmSens, Palm Instruments, Niederlande) angeschlossen. Bevor verschiedene Glukosekonzentrationen gemessen wurden, wurde ein Voltammogramm aufgenommen, um das Arbeitspotential im Plateau zu bestimmen. Zur Glukosemessung wurde eine Spannung von +600 mV an die Arbeitselektrode vs. die Ag/AgCl-Referenzelektrode angelegt. Der generierte Strom bei Veränderung der Glukosekonzentrationen wurde in Abhängigkeit von der Zeit aufgenommen. Jede Glukosekonzentration im Bereich von $0,5 \mathrm{mM}$ bis $3 \mathrm{mM}$ wurde unter ständigem Rühren der Substratlösung für 10 min gemessen. 
Der Degradationsprozess des Sensorchips wurde unter laminaren Strömungsbedingungen beurteilt. Zunächst wurde jeder Chip unter UV-Licht sterilisiert und jeweils in eine Einzelkammer einer Mikrotiterplatte gelegt. 0,01 g des Enzyms Protease (Streptomyces griseus, Type XIV, $\geq 3,5 \mathrm{U} / \mathrm{mg}$ Pulver, SigmaAldrich, St. Louis, USA) wurde in $10 \mathrm{~mL}$ PBS Puffer (phosphatgepufferte Salzlösungstablette, Biotechnology Grade, VWR International, Radnor, USA) gelöst. Die einzelnen Kammern der Mikrotiterplatte wurden jeweils mit Enzymlösung gefüllt, bis jeder Sensorchip komplett mit Lösung bedeckt war. Die Mikrotiterplatte wurde verschlossen, mit 70\%-Ethanol von außen gereinigt und in einen Inkubator (Heracell $150 \mathrm{i} \mathrm{CO}_{2}$ incubator, Thermo Scientific, Waltham, USA) bei $37^{\circ} \mathrm{C}$ gelegt. Nach 2, 4 und 10 Tagen wurde die Mikrotiterplatte vorsichtig herausgenommen.

\section{Ergebnisse}

Morphologische Charakterisierung der Platin-
elektrode auf dem Fibroinsubstrat

Die mittels Dünnschichttechnologie hergestellten PtElektroden auf dem Fibroinsubstrat wurden mittels Rasterelektronenmikroskopie (REM, JEOL JSM$7800 \mathrm{~F}$, Deutschland) charakterisiert. Dabei wurde eine Beschleunigungsspannung von $50 \mathrm{kV}$ angelegt. Abb. 3 a) zeigt die homogene Platinschicht ohne Risse auf dem Fibroinsubstrat. Die Platinstruktur wird zudem bei den EDS-Messungen (Energiedispersive Röntgenspektroskopie, JEOL JSM-7800 F, Deutschland) in Abb. 3 b) sichtbar. Platin ist hier in Magenta eingefärbt und der Kohlenstoff des Fibroinsubstrats in Gelb.
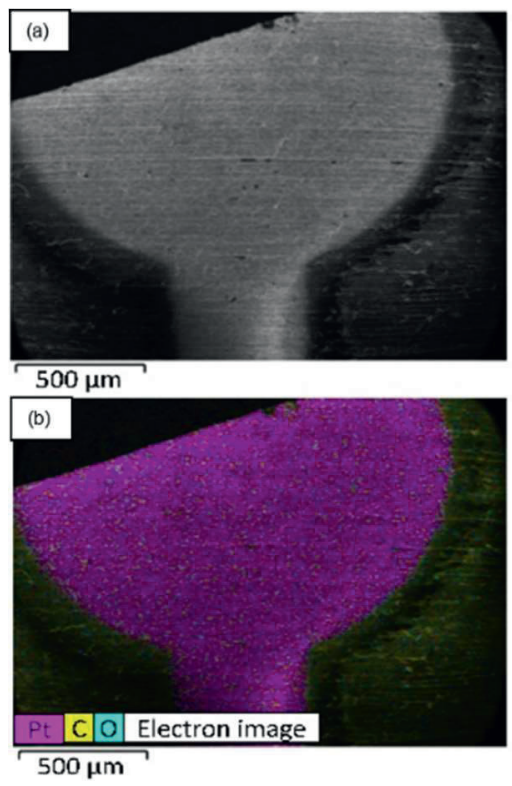

Abb. 3: REM-Aufnahmen der Pt-Arbeitselektrode auf dem Fibroinsubstrat bei einer Vergrößerung von 1000x (a) und das entsprechende EDS-Bild (b).

\section{Elektrochemische Charakterisierung des biokompa- tiblen Glukosebiosensors}

Die Sensitivität des hergestellten biokompatiblen Glukosebiosensors wurde amperometrisch untersucht. Dazu wurden verschiedene Glukosekonzentrationen zwischen $0,5 \mathrm{mM}$ und $3 \mathrm{mM}$ in Phosphatpuffer bei einem pH Wert von 7,4 nacheinander gemessen. Zur statistischen Auswertung wurden diese Messungen drei Mal wiederholt. In Abb. 4 ist exemplarisch eine entsprechende Kalibiergerade in Blau dargestellt. Es konnte eine Sensitivität von $0,2 \pm 0,01 \mu \mathrm{A} / \mathrm{mM}$ über den gesamten gemessenen Konzentrationsbereich erreicht werden.

Zusätzlich wurden die Eigenschaften des Sensors überprüft, inwiefern blutähnliche Komponenten einen Einfluss auf die Sensorcharakteristika haben. Dazu wurden die gleichen Glukosekonzentrationen in Ringerlösung $(\mathrm{pH} 7,4)$ gemessen, welche analoge Elektrolytkonzentrationen wie Blut enthält. Die entsprechende beispielhafte Kalibiergerade ist ebenfalls in Abb. 4 (schwarze Kurve) zu sehen. Es konnte eine vergleichbare Sensitivität mit $0,16 \pm 0,01 \mu \mathrm{A} / \mathrm{mM}$ über den gesamten Messbereich erzielt werden. Die beiden Experimente zeigen, dass der hergestellte biokompatible Glukosebiosensor erfolgreich verschiedene Glukosekonzentrationen messen kann und großes Potential hat, um in realen Proben angewendet zu werden.

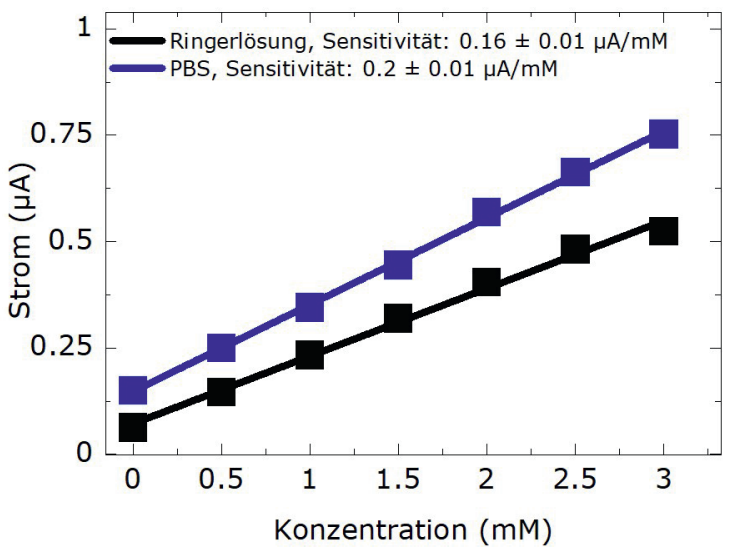

Abb. 4: Kalibiergeraden des biokompatiblen Glukosebiosensors mit dem immobilisierten Enzym GOX gemessen in PBS (blau) und in Ringerlösung (schwarz) mit verschiedenen Glukosekonzentrationen zwischen 0,5 mM und $3 \mathrm{mM}$. PBS: Phosphatgepufferte Salzlösung.

\section{Beurteilung der Degradation der Platinelektrode abgeschieden auf dem Fibroinsubstrat}

Die Degradationstests der Pt-Elektroden (abgeschieden auf dem Fibroinsubstrat) ergaben, dass beide Materialien über einen Zeitraum von 10 Tagen, gelagert in einer Enzym-Pufferlösung, fast vollständig degradieren. In Abb. 5 wird eine lichtmikroskopische 
Aufnahme an Tag 0 gezeigt, bei der deutlich die vier Pt-Elektroden auf dem Fibroinsubstrat zu erkennen sind. Die Aufnahme an Tag 10 zeigt hingegen nur noch staubähnliche Partikel.

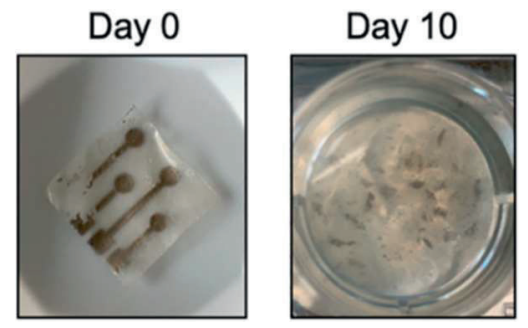

Abb. 5: Degradationstests des Fibroinsubstrats mit den abgeschiedenen Pt-Dünnschichtelektroden über einen Zeitraum von 10 Tagen.

\section{Diskussion}

Es wurde ein flexibler biokompatibler Glukosebiosensorchip mit integrierter Pt-Arbeits-, Pt-Gegen- und $\mathrm{Ag} / \mathrm{AgCl}$-Quasi-Referenzelektrode beschrieben. Zur Herstellung der Elektrodenstrukturen wurde die Kombination aus Dickschicht- und Dünnschichttechnologien angewendet.

Die Besonderheit des Biosensorchips lag in der Substratstruktur, die aus dem Seidenfibroin des Bombyx mori hergestellt wurde. Dieses biokompatible und biodegradierbare tierische Naturprodukt kann künstliche Materialien ersetzen und hat damit ein hohes Potential bei der Herstellung von "Green Electronics" zur Anwendung beispielsweise in der Lebensmittelindustrie oder bei biomedizinischen Implantaten.

Der entwickelte Glukosebiosensorchip wurde elektrochemisch hinsichtlich seiner Sensitivität charakterisiert. Dazu wurden Glukosekonzentrationen im Bereich von $0,5 \mathrm{mM}$ bis $3 \mathrm{mM}$ in Pufferlösung bei einem $\mathrm{pH}$ Wert von $\mathrm{pH} 7,4$, entsprechend dem $\mathrm{pH}-$ Wert von Blut, gemessen. Die gleichen Messungen wurden in Ringerlösung wiederholt. Die Sensitivitätswerte zeigen, dass der Biosensorchip gute Voraussetzungen besitzt, um zukünftig in realen Proben, wie zum Beispiel in Blut oder Urin getestet zu werden. Im nächsten Schritt muss der entwickelte Glukosebiosensor weiter verbessert werden, um Glukosekonzentrationen im medizinisch relevanten Bereich nachweisen zu können. Ein Ansatz ist hier die Verbesserung der Enzymmembran, sowie die Immobilisierungsmethode.

Die Degradationstests des Fibroinsubstrats mit integrierten Pt-Dünnschichtelektroden zeigen, dass sich der Sensor nach 10 Tagen fast vollständig aufgelöst hat. Damit schafft der Biosensor gute Voraussetzungen, um im Körper eingesetzt werden zu können.

Zukünftig soll dieser Ansatz weiterentwickelt werden, so dass der Biosensorchip gezielt zu einem bestimmten Zeitpunkt degradiert. Dies wäre bei einem in-vivo Einsatz von Vorteil, so dass der verwendete Biosensor nicht zu einem späteren Zeitpunkt wieder operativ entfernt werden müsste.

\section{Literatur}

[1] MOHANKUMAR, P., AJAYAN, J., MOHANRAJ, T., YASODHARAN, R., Recent developments in biosensors for healthcare and biomedical applications: A review. Measurement, 167 (2021), 108293

[2] ZHU, B., WANG, H., LEOW, W.R., CAI, Y., LOH, X.J., HAN, M.-Y., CHEN, X., Silk fibroin for flexible electronic devices. Adv. Mater., 28 (2016), Nr. 22, S. 4250-4265

[3] VEPARI, C., KAPLAN, D.L., Silk as a biomaterial. Prog. Polym. Sci., 32 (2007), Nr. 8-9, S. 991-1007

[4] SEVES, A., ROMANO, M., MAIFRENI, T., SORA, S., CIFERRI, O., The microbial degradation of silk: A laboratory investigation. Int. Biodeterior., 42 (1998), Nr. 4, S. 203-211

[5] LUCAS, F., SHAW, J.T.B., SMITH, S.G., The amino acid sequence in a fraction of the fibroin of Bombyx mori. Biochem. J., 3 (1957), Nr. 66, S. $468-479$

[6] ARAI, T., FREDDI, G., INNOCENTI, R., TSUKADA, M., Biodegradation of Bombyx mori silk fibroin fibers and films. J. Appl. Polym. Sci., 91 (2004), Nr. 4, S. 2383-2390

[7] MOLINNUS, D., DRINIC, A., IKEN, H., KRÖGER, N., ZINSER, M., SMEETS, R., KÖPF, M., KOPP, A., SCHÖNING, M.J., Towards a flexible electrochemical biosensor fabricated from biocompatible Bombyx mori silk. Biosens. Bioelectron., 183 (2021), 113204

[8] MOLINNUS, D., BÄCKER, M., IKEN, H., POGHOSSIAN, A., KEUSGEN, M., SCHÖNING, M.J., Concept for a biomolecular logic chip with an integrated sensor and actuator function. Phys. Status Solidi A, 2012 (2015), Nr. 6, S. 1382-1388

\section{Danksagung}

Die Autoren danken D. Rolka für die REM- und EDSAufnahmen, sowie S. Fuest, A. Baranowsky, J. Kiesel und $S$. Woege für deren Unterstützung bei der Durchführung von einzelnen Experimenten. 